\title{
La mujer campesina en la agricultura y seguridad alimentaria
}

Sulema LOAYZAALATRISTA

\section{RESUMEN}

Se analiza la participación de la mujer campesina en la actividad agropecuaria alimentaria, su relación y desenvolvimiento en el mercado y su protagonismo en las estrategias de seguridad alimentaria de la pequeña agricultora del sur andino, que constituye uno de los más importantes sectores proveedores de alimentos para la población, en el contexto del comercio mundial y del boom de la comida andina. El estudio se ha desarrollado en comunidades campesinas de policultivos de valles interandinos de Arequipa y Cusco.

PALABRAS CLAVE: Mujer y pequeña agricultura, mujer campesina, estrategias agropecurias y seguridad alimentaria.

\section{Rural women in agriculture and food security}

\section{ABSTRACT}

Here we analyze female peasants participation in agricultural and food activities, their relationship and involvement in the market, protagonist role on food security strategies of small farmer communities in the south part of the Andes, which is one of the main food providers for the communities, in the context of world commerce and the economic boom of Andean food. This study was done with peasant communities, farming multiple crops, in the Andean valleys of Arequipa and Cusco.

KEYWORDS: Women, small farmers, female peasants, agricultural strategies, food security. 


\section{Marco teórico y aspectos generales}

a agricultura familiar es una forma de vida y de cultura que tiene como objetivo la reproducción social de la familia y la comunidad, gestiona sistemas productivos agropecuarios de pequeña escala con mano de obra predominantemente familiar no asalariada, para producir principalmente alimentos y complementariamente otros bienes y servicios, cuyo destino final es el mercado y el autoconsumo, característica por la que en el Perú y en los países de la región se la denomina también agricultura alimentaria.

La pequeña agricultura familiar se desenvuelve en interrelación dinámica con su entorno social, económico, político, cultural y ambiental. Demanda innovación y adaptación constante, en respuesta a los condicionantes del mercado y del entorno social, como también a las fluctuaciones del medio físico natural, particularmente por efecto del cambio climático. Es inseparable de la unidad familiar, dado que cuenta con los mismos recursos y las decisiones en su manejo influyen tanto en la familia como en la unidad productiva. Por ello, la agricultura familiar busca primero minimizar los riesgos antes de maximizar sus ingresos económicos.

Los agricultores familiares gracias a su gran diversidad, número y cercanía a los consumidores proveen la mayor parte de los alimentos para el mundo. Ello los convierte en garantes de la seguridad alimentaria y nutricional a nivel local, regional, nacional e internacional, su producción contribuye a garantizar el derecho humano a una alimentación adecuada. ${ }^{1}$

La pequeña agricultura familiar es un sistema de producción de vital importancia en el actual contexto internacional de creciente dependencia de los mercados globales. Según la FAO, en América Latina y el Caribe representa el $80 \%$ de los sistemas de producción, aporta entre el 30\% y 40\% del PBI agrícola regional, además incorpora más de $60 \%$ del empleo rural, razón por la cual constituye un pilar fundamental para avanzar hacia el desarrollo equilibrado de los territorios y sociedades rurales. ${ }^{2}$

En el Perú, el minifundio es el subsector que predomina en la producción de alimentos, $1^{-} 811,000$ unidades agropecuarias menores de 5 Has., que en promedio alcanzan $3.1 \mathrm{Has}$. cada una, representan el $82 \%$ del total de unidades productivas agrarias, que pueden considerarse de pequeños propietarios agrarios. Geográficamente se distribuyen, $15 \%$ en la Costa, $14 \%$ en la Selva y una gran mayoría, de $71 \%$ de las mismas, en la sierra. Dichas unidades

FAO. Cumbre Mundial de la Alimentación. 1996

FAO - BID. Políticas para la Agricultura Familiar en América Latina y el Caribe. 2007.

\section{0/ REVISTA DESOCIOLOGIA 25}


productivas, que incluyen a las Comunidades Campesina y Comunidades Indígenas, generan el $80 \%$ de los productos alimenticios que consume el mercado nacional, a través de los centros de abastos de costa, sierra y selva. ${ }^{3}$ Contribuyen para que la población nacional logre una alimentación más autónoma, no sólo en cantidad sino en calidad. A lo cual se agrega la diversidad y el valor nutricional de los alimentos en la mesa de la familia peruana, que se debe casi exclusivamente a la agricultura familiar, considerando que las importaciones y la producción agroindustrial se restringen a pocos renglones.

La región de la sierra peruana reúne a la gran mayoría de las pequeñas unidades agropecuarias, productoras de alimentos del país. Es relevante así mismo que determinados sectores de las mismas vienen incursionando en el cultivo de productos alimenticios andinos que destinan para la exportación, tales como la quinua y la kiwicha, que vienen siendo reconocidos en los mercados del mundo por sus cualidades nutritivas y formas de manejo productivo sostenibles, amigables con el entorno ecológico y social. Hace más de una década los medios sociales de comunicación vienen protagonizando campañas que dan a conocer las bondades gastronómicas y nutritivas de la comida peruana, a la par que divulgan la importancia de la agricultura alimentaria andina, como depositaria de un acervo tecnológico de diversidad de cultivos nativos y exóticos destinados a la alimentación humana, sustento en que reposa el boom de la gastronomía peruana.

La agricultura familiar en el Perú guarda la principal reserva genética de la diversidad de cultivos andinos, convirtiéndose en el principal sistema de producción que garantiza la protección de nuestra biodiversidad ecológica y genética, recurso potencial con vista a las necesidades de desarrollo productivo para atender la creciente demanda de alimentos en el mundo.

Durante las últimas décadas se viene operando en muchas regiones y países la tendencia denominada "feminización de la agricultura", que se caracteriza por el creciente dominio de las mujeres en la producción agrícola, aparejado por la disminución de la presencia de varones en la actividad. Dicha evolución es acompañada por el incremento de hogares encabezados por mujeres, debido a la emigración masculina, conflictos armados, abandono y creciente número de madres solteras. Si bien las estadísticas oficiales de América Latina y El Caribe colocan el porcentaje de hogares encabezados por mujeres en un $17 \%$, un estudio realizado por el FICA, indica que esa proporción era mucho más alta, así en el caso del Perú era de 43\%.4 Para el año 2012, el

3 INEI. IV Censo Nacional Agropecuario. 2012.

4 Instituto Interamericano de Cooperación Agrícola - FICA. 1994 
INEI refiere en las estadísticas del censo agropecuario que un 30\% de unidades agropecuarias del Perú están a cargo de mujeres. ${ }^{5} \mathrm{~A}$ lo anotado cabe agregar que las cifras oficiales infravaloran significativamente la proporción de mujeres que poseen y administran explotaciones agrícolas. Muchas veces se supone o se da como legalmente reconocido que el varón es el "jefe de hogar" y que también es el responsable de la explotación, aun cuando es la mujer quien se ocupa de las actividades cotidianas y decisiones en el manejo productivo de las pequeñas explotaciones agrícolas, productoras de alimentos.

Las mujeres campesinas se han responsabilizado durante siglos de las tareas domésticas, del cuidado y preservación del alimento de sus familias, del cultivo para el autoconsumo y la comercialización de sus excedentes, cargando con el trabajo reproductivo y productivo, pero lo hacen en condiciones de inequidad y de una manera invisible en las cuentas nacionales, ocupando una esfera privada en la sociedad. En cambio, las principales transacciones económicas familiares son todavía llevadas a cabo por los varones, ocupando la esfera pública y oficial. Esta división de roles mantiene los papeles asignados como masculinos y femeninos, que aún hoy, con ligeras variaciones, perduran en nuestras sociedades.

Las mujeres rurales desempeñan un papel fundamental en las economías agrícolas de la mayor parte del mundo, participan en la producción de cultivos, el cuidado del ganado, la provisión de agua, de alimentos y combustible para sus familias, así mismo, lo hacen en actividades no agrícolas para diversificar los medios de subsistencia, las mujeres rurales son también guardianas de la biodiversidad y de las semillas, defienden la biodiversidad, como parte de su relación con la madre tierra. Según la FAO, en los países del hemisferio sur, las mujeres producen entre $60 \%$ y $80 \%$ de los alimentos y un $50 \%$ a nivel mundial. Ellas son las principales productoras de alimentos básicos, como el arroz, trigo, papas y maíz, que alimentan a las poblaciones más empobrecidas del sur global, sin embargo, son ellas junto con los niños los más afectados por el hambre. En dichos países, según la misma fuente, la población femenina económicamente activa en la agricultura se mantiene por encima del 50\% y su contribución directa en el cultivo y producción de alimentos sigue siendo relevante a nivel mundial. ${ }^{6}$ En América Latina y el Caribe se observa, como patrón constante que, en promedio, un $78 \%$ de mujeres están incorporadas de manera activa al trabajo agrícola entre los 15 y los 59 años de edad, constituyen una de las fuerzas que mueven la economía de los territorios y son

5 Instituto Nacional de Estadística - INEI. IV Censo Nacional Agropecuario. 2012.

6 García, Z. 2006.

\section{2/ REVISTA DESOCIOLOGÍA 25}


corresponsables de su desarrollo, así mismo, son guardianas de la estabilidad de las familias rurales. Esta doble tarea les confiere un carácter estratégico para el desarrollo y bienestar rural, cuyo potencial se comprueba con el análisis de su contribución pluriactiva.

Las mujeres de las pequeñas unidades agropecuarias dedican más de ocho horas diarias a las tareas productivas, trabajando intensivamente en los cultivos y la crianza de animales, pero adicionalmente realizan las labores domésticas familiares, la comercialización de sus productos en ferias y mercados y, en muchos casos, se contratan como asalariadas en otros predios agrícolas y realizan la venta de ambulatoria de comidas. Dicha participación de la mujer en la agricultura familiar aporta a la seguridad alimentaria y a la generación de ingresos económicos.

La agricultura familiar reproduce todavía un sistema patriarcal inequitativo de la organización familiar, dentro del cual las mujeres a menudo se encuentran en situación de dependencia y sobrecarga de trabajo. Las brechas de inequidad se manifiestan a temprana edad entre varones y mujeres, siendo la limitación en el acceso a la educación de las mujeres el factor que más negativamente incide en su formación y las perspectivas personales de cada mujer, limitando a su vez su acceso a los mercados laborales, los salarios, la capacitación técnica y profesional y consiguientemente, se limita también su acceso a los activos productivos (tierra, créditos, tecnología, etc.). Las mujeres de los pequeñas unidades agropecuarias tienen en promedio un bajo nivel de escolaridad, en el Perú no superan cuatro años de educación primaria y todavía prevalecen entre ellas altos niveles de analfabetismo, limitando su acceso a las innovaciones tecnológicas.

Si bien el rol productivo de la mujer es predominante en la agricultura alimentaria del Perú, no lo es necesariamente de poder en la toma de decisiones, inequidad que sigue siendo un problema intrínseco de la sociedad en general. A nivel nacional, un $28 \%$ de las mujeres agropecuarias no fue a la escuela, el triple de lo que se registra entre los hombres. La FAO estima que si las mujeres del mundo tuvieran el mismo acceso a los recursos que los hombres, podrían liberar de 100 a 150 millones de personas del hambre. Ello implica promover la visibilidad de la participación productiva de la mujer de la agricultura familiar y su contribución al desarrollo económico, social, cultural y medio ambiental.

El crecimiento económico sostenido del Perú de la década pasada ha sido desigual, así por ejemplo, el año 2012 el PBI del país creció en 6.3\%, sin embargo la producción agropecuaria creció sólo 5.1\%; comportamiento 
que fue un común denominador durante dichos años, haciendo que el peso de la actividad agropecuaria disminuya, siendo su contribución actual 7.2\% del PBI nacional. Sin embargo, los aportes más significativos del agro se dan en el campo (32\%) y en el abastecimiento de productos alimenticios (90\%). Según Cavassa y Mescler, la relevancia de la pequeña agricultura en el Perú se mantiene por: a) el constante crecimiento de la población rural, b) la predominancia de la PEA agropecuaria en varias regiones de la sierra y c) la abrumadora mayoría de la pequeña agricultura en el paisaje rural del país. ${ }^{7}$ En dicho sentido, cabe señalar que el $24 \%$ de la PEA sigue siendo rural. Entre los cambios orientados en la última década a promover la inclusión productiva de los pequeños agricultores como una forma de lucha contra la pobreza, el gasto realizado no muestra una buena performance, comparado con otros sectores, la dinámica de las inversiones públicas para la pequeña agricultura tiene poca relación con las políticas nacionales del sector agrario.

La agricultura familiar, constituida principalmente por pequeñas unidades productivas, generalmente en predios agrícolas menores de una hectárea de extensión, conducidas por miembros de familia, de escasos recursos materiales y tecnológicos y aún con el redoblado trabajo agrícola y de comercialización que realiza la mujer campesina, es especialmente vulnerable a los efectos de la globalización, una de cuyas manifestaciones es la volatilidad de los precios internacionales, que no permiten planificar a largo plazo o encontrar reservas que amortigüen sus impactos, exponiendo a riesgos imprevisibles a los agricultores, amenazando su estabilidad y la seguridad alimentaria y nutricional de la población y la conservación in situ de la agro biodiversidad.

Así mismo, la creciente variabilidad de los fenómenos provocada por el cambio climático está afectando la compleja relación que existe entre seguridad alimentaria, la producción agraria y el comercio. Los efectos del cambio climático atentan contra las capacidades de adaptación inherentes de la agricultura familiar, pudiendo perjudicar sus producciones y existencia, con efectos negativos en la seguridad alimentaria local y nacional. ${ }^{8}$ Las mujeres rurales, las principales actoras en las tareas de afrontar la seguridad alimentaria, se ven muchas veces enfrentadas a las pérdidas de sus cosechas, debido a sequías o inundaciones.

La pequeña agricultura en el Perú se desenvuelve en las áreas rurales, donde la incidencia de la pobreza en la población persiste todavía con caracteres

7 Cavassa, Augusto y Mescler, Evelyn. Actividades Agropecuarias en el Campo Peruano. 2012.

8 Piñeiro, Martín. Las relaciones entre seguridad alimentaria, cambio climático y comercio internacional. Paper № 146. Buenos Aires. 2011.

\section{4/ REVISTA DESOCIOLOGÍA 25}


graves. Si bien a nivel nacional y particularmente en los sectores urbanos las campañas sociales iniciadas hace más de diez años tuvieron respuesta favorable, habiéndose logrado reducir la pobreza a $25.8 \%$ al año 2012. En el ámbito rural, no obstante, no son perceptibles cambios significativos, registrándose una incidencia de $53.0 \%$ de pobreza el mismo año. ${ }^{9}$ Cuadro que se agrava por la persistencia significativa de pobreza extrema (19.7\%) en la población rural del país. En el sector agropecuario, descontando los problemas de la estacionalidad, el fortalecimiento de la canasta a favor se ha estancado, lo que hablaría de un impacto negativo del comercio internacional. ${ }^{10}$ La pobreza, medida en la práctica por la canasta básica de consumo, resulta de multiplicar el costo de la canasta básica personal por el número de miembros de una familia, límite que muchas familias rurales del país no están en condiciones de alcanzar. Así la relación de la seguridad alimentaria con la pobreza tiene un orden reversible de causa-efecto, debido a que la pobreza puede determinar la inseguridad alimentaria, pero que también puede ser el efecto de la misma.

La seguridad alimentaria es un enfoque multidimensional aplicable a nivel del individuo, hogar, nación y global, que se consigue cuando todas las personas, en todo momento tienen acceso físico y económico a suficientes alimentos seguros y nutritivos para satisfacer sus necesidades alimenticias y sus preferencias con el objetivo de llevar una vida activa y sana. ${ }^{11}$ La seguridad alimentaria incluye: 1) La disponibilidad de alimentos asociado al incremento de los niveles de producción en las zonas de mayor vulnerabilidad. 2) El uso de los alimentos, enfocado desde la inocuidad de los mismos, su manipulación y aprovechamiento biológico. 3) La estabilidad del suministro, que tiene que ver con el empleo y la disponibilidad de alimentos.

Los problemas persistentes en el agro peruano, sumados a la desigualdad social que atraviesa el país, ocasionan que las familias peruanas de las zonas rurales estén expuestas a la inseguridad alimentaria, al afrontar dificultades en el acceso, disponibilidad, utilización y permanencia de los alimentos.

9 INEI. Nota de Prensa № 067 - 08 de Mayo 2013. El INEI mide la pobreza monetaria comparando los gastos de los hogares con la línea de pobreza. Dicha línea es aquella que permite adquirir una Canasta Básica de Consumo suficiente para satisfacer los requerimientos nutricionales (alimentos) y otras necesidades básicas de los hogares (educación, vestido, salud, transporte, entre otros). El costo promedio de la Canasta Básica de Consumo se estima en S/ 260 mensuales por persona a nivel nacional, S/. 284 por persona en las zonas urbanas y S/. 198 soles para las zonas rurales. Aquellas personas que tengan un gasto por debajo de dichos montos, según cada caso, serían consideradas pobres.

10 CEPES. 2013.

11 FAO. Cumbre Mundial de la Alimentación. 1996. 


\section{Agricultura alimentaria}

La pequeña unidad productiva agropecuaria, caracterizada en el Perú por su vocación productiva de alimentos, se compone esencialmente de la familia, como elemento dinamizador del conjunto y como proveedor de mano de obra, los recursos naturales de tierra y agua, los recursos económicos de animales, productos agropecuarios, semillas e insumos, herramientas y máquinas. La familia como actor central, distribuye entre sus miembros las diferentes funciones complementarias entre sí, en una organización donde el rol que la mujer desempeña es fundamental. Las mujeres participan en todas las etapas de la agricultura, desde la siembra hasta la cosecha y en los procesos agroindustriales y la comercialización de los productos alimentarios, tienen un papel protagónico en la producción de pequeñas especies y si se trata de producción animal a gran escala son responsables del suministro de agua, de la higiene de los establos y de la ordeña.

La familia decide cómo emplear los recursos, su disposición para las necesidades de sus miembros o su aprovechamiento en el sistema productivo. Este nexo entre la familia, la chacra y los recursos distinguen a estas pequeñas unidades productivas de otras formas de explotación agropecuaria. Las familias se relacionan entre sí, intercambiando recursos y productos, apoyándose mutuamente o agrupándose para el manejo de organizaciones productivas mayores, como se verá en la producción tecnificada de cuyes y también para el desarrollo o mantenimiento de infraestructura agrícola, como tradicionalmente se hace con los sistemas de riego.

El cuidado de la parcela familiar y la crianza de animales domésticos menores son actividades que pueden alcanzar mucho éxito en la medida que contribuyan en su desarrollo un mayor número de miembros de la familia, cuando no ocurre así, aquellas actividades sobrecargan las responsabilidades de trabajo de la ama de casa, tanto dentro como fuera del hogar, ya que el tiempo dedicado al mantenimiento de la parcela familiar y de la crianza entra en competencia con el tiempo que se quiere dedicar a otras tareas. En efecto, los niveles de nutrición del hogar son asociados al acceso de la mujer al ingreso y la toma de decisiones en materia de gasto familiar. La falta de acceso y control de las mujeres sobre los recursos económicos limitan el ejercicio de su autonomía y participación en la toma de decisiones. ${ }^{12}$

\section{6/ REVIISTA DE SOCIOLOGÍA 25}


Además de los papeles decisivos en la producción de alimentos, las mujeres campesinas contribuyen a la seguridad alimentaria de otros modos importantes: como aquellos que preservan la biodiversidad y los recursos fito genéticos, ampliamente reconocidos como factores esenciales para la seguridad alimentaria. Al ser las responsables de la producción de alimentos y cuidados de la familia, las mujeres del campo conservan los conocimientos tradicionales del valor y la diversidad de usos de plantas para la nutrición y la salud, suelen experimentar y adaptar especies indígenas, a menudo son las guardianas del tradicional conocimiento de las plantas nativas.

Los Andes en el Perú constituyen un sistema con una agricultura de alta montaña, un medio ecológico altamente variado, donde la seguridad alimentaria se basa en el uso adecuado de la biodiversidad. Las características de la población campesina andina a la que pertenecen las mujeres en estudio y el entorno socioeconómico de los sistemas agrícolas donde actúan permiten explicar a cabalidad el papel desempeñado por la mujer campesina andina y su relación con la conservación, evolución y el uso potencial de los recursos fito genéticos andinos. Entre los cultivos que la mujer campesina en el Perú ha participado en domesticar y que actualmente maneja en los Andes, destacan por su importancia para la seguridad de la alimentación en el mundo el maíz y las variedades de papas que han recibido atención de la investigación agrícola occidental. ${ }^{13}$ Sin embargo y para adecuar el nivel nutricional de la dieta de su familia, la mujer campesina ha sabido mantener en la producción agrícola otras especies que, además de diversificar la alimentación familiar, permiten un uso más racional e intensivo de la chacra.

Con la finalidad de llevar a cabo el estudio propuesto hemos seleccionado dos provincias de la sierra sur, una, La Unión Cotahuasi, en el departamento de Arequipa y la otra, Quispicanchis, en el Cusco, que albergan áreas rurales emplazadas en valles interandinos, caracterizadas por su vocación agro alimentaria de pequeños productores, que estimamos son representativas de los modos de producción agropecuaria del sur andino.

Tanto en Quispicanchis como en La Unión, un promedio de $72 \%$ de las mujeres encuestadas tienen familia constituida, con pareja, mayormente casadas y también convivientes. Las mujeres separadas y viudas son minorías entre $12 \%$ y $16 \%$, respectivamente. En Quispicanchis un $70 \%$ de las mujeres tienen edades que fluctúan entre 26 y 45 años y $30 \%$, entre 46 años y 64 años. En La Unión el 50.0\% de mujeres están dentro del grupo etario de 46

De La Torre, Ana. 1988. 
a 64 años, $30 \%$ entre 26 años y 45 años y dos grupos minoritarios, de 10\% cada uno, de jóvenes de 18 a 25 años de edad y otro de la tercera edad, de 65 y más años. En Quispicanchis y La Unión los cónyuges tienen en promedio mayores edades que las mujeres, con la excepción de que en La Unión no hay cónyuges con más de 65 años de edad.

El nivel de estudios alcanzado en promedio por las encuestadas es bajo, en ambas provincias, un $10 \%$ de mujeres son analfabetas y alrededor del $60 \%$ de las mismas sólo tienen educación primaria. Una minoría de 30\% de las encuestadas tienen secundaria completa. El nivel de estudios del cónyuge es un poco más elevado, no existe analfabetos entre ellos y algo más del 50\% tienen secundaria completa, no obstante, apenas $2.9 \%$ de los cónyuges ha alcanzado nivel de estudios superior técnico. El número promedio de hijos es de 3.8 y el número promedio de hijos residentes en casa es de 2.1, es decir, de cada 4 hijos, 2 de ellos viven en casa y 2 viven alajados, por motivos relativos a estudio, trabajo y la formación de un nuevo hogar. En general, constituyen familias que en promedio son pequeñas, con no más de cuatro miembros residentes en el hogar, dado que los cónyuges o convivientes en gran medida tienen ocupaciones que los alejan del predio familiar.

En ambas provincias, una mayoría absoluta de $76 \%$ de mujeres encuestadas tienen por ocupación principal la agricultura, generalmente asociada a las ocupaciones de ama de casa, manteniéndose permanentemente en contacto con el predio agrícola. Una minoría de $22 \%$ de las mismas son agricultoras y comerciantes, generalmente de pequeños negocios en sus mismas localidades. Entre los cónyuges varones, un 44\% tienen por ocupación principal la agricultura, algunos asociados a la ocupación de comerciante y en menor proporción como albañiles. Adicionalmente, un $47 \%$ de cónyuges son transportistas, empleados y jornaleros, fuera de sus localidades.

Entre los principales recursos de estos agricultores alimentarios sur andinos se cuentan sus pequeñas tierras y el ganado y animales menores de su crianza. Las tierras agrícolas en ambas provincias tienen un tamaño promedio de $1.1 \mathrm{Has}$, siendo algo mas grandes las de La Unión; generalmente repartidas en dos o más parcelas, que en su gran mayoría disponen de agua de riego. Un sector minoritario de las agricultoras manejan adicionalmente parcelas alquiladas, más pequeñas. El ganado de las entrevistadas se compone principalmente de pocas cabezas de ganado vacuno, en promedio 3.1 cabezas en Quispicanchis y 2.6 en La Unión y de porcinos, en promedio 2.9 cabezas en Quispicanchis y 1.8 cabezas en La Unión. La cantidad de animales vendidos o beneficiados en el transcurso de un año aproximadamente equivale a la mitad

\section{8/ REVISTA DE SOCIOLOGÍA 25}


de la tenencia del ganado vacuno y en el caso de los porcinos es una cantidad casi equivalente al total de la tenencia, en ambas provincias.

En la tenencia de animales menores destaca la de cuyes entre las entrevistadas de Quispicanchis, al haberse desplegado en los últimos años adoptando características de pequeñas granjas tecnificadas, con poblaciones promedio de 130 cuyes de cada una y cuya venta anual es casi equivalente a dicha cantidad. En La Unión, el promedio de cuyes de cada entrevistada es de 18 animales. La tenencia de gallinas cobra relativa importancia en ambas provincias, con un promedio de 5.9 animales, que en Quispicanchis destinan preferentemente a la venta, mientras que en La Unión más auto consumen. En esta última provincia cobra relativa importancia la crianza de ovinos, que alcanza a un promedio de 11 animales por cada entrevistada, preferentemente destinados al autoconsumo.

La actual estructura productiva de cultivos en los predios familiares denota un notable auge de las extensiones cultivadas de kiwicha y quinua, particularmente en la provincia de La Unión, donde a la kiwicha se destina las mayores áreas, que en promedio alcanzan a 0.62 Has por unidad productiva y en Quispicanchis a 0.25 Has. Los cultivos de quinua en La Unión tienen en promedio 0.33 Has. y en Quispicanchis 0.15 Has. Los cultivos de maíz, cebada, papas, arvejas y habas mantienen su importancia tradicional, con áreas promedio cultivadas en ambas provincias entre 0.37 Has. y 0.25 Has. Los cultivos de trigo son algo menores, con un promedio de $0.21 \mathrm{Has}$. por unidad productiva, siendo, en general, mayores las extensiones de los cultivos de trigo en la Unión. Mantienen relativa importancia los cultivos de alfalfa, hortalizas y tarwi, en extensiones promedio por familia entre 0.25 Has. y 0.19 Has.

Es preciso poner de relieve que cada uno de los diversos cultivos en manos de estos pequeños agricultores cumple una función determinada, según las concepciones culturales de sus protagonistas, que aprecian los factores físicos y ambientales, la disponibilidad de agua, piso ecológico, aptitud de las tierras, las exigencias de cada cultivo, su estacionalidad, la demanda del mercado y las necesidades económicas y alimentarias familiares, que generalmente es la mujer campesina quien evalúa cada situación y adopta las acciones más adecuadas para su familia.

El análisis relativo a las formas de participación de los miembros de familia en las actividades productivas de la pequeña agricultura nos muestra el panorama que se muestra a continuación. Las mujeres en las provincias de Quispicanchis y de La Unión tienen una activa participación en labores de cultivo según tipo de labor, en muchos casos, definidamente superiores a 
la participación de los hombres. Así, encontramos que la mujer tiene mayor participación en las labores de preparación de semillas, riego y deshierbo de cultivos y en la cosecha y almacenaje de productos, en proporciones que oscilan entre $69 \%$ de encuestadas (preparación de semillas) y 92\% de encuestadas (deshierbo). También se dan labores que realizan en forma principalmente mancomunada entre la entrevistada y su cónyuge, como el barbecho $(52 \%$ de casos) y la siembra (61\% de casos). La aplicación de insecticidas la realizan aproximadamente en similares proporciones las parejas en ambas provincias, aunque en La Unión hay una relativa mayor presencia de la mujer. El arado de la tierra es realizado en un $42 \%$ de casos por el cónyuge y en $39 \%$ de casos en forma compartida por las parejas.

La participación productiva de la mujer también se manifiesta con caracteres relevantes bajo la óptica del tipo de cultivos, así en el caso de las hortalizas, verduras y flores lo realizan $96 \%$ de las entrevistadas, los cultivos de menestras, como habas y arvejas los realizan $80 \%$ de entrevistas, cobra un peso mayoritario de $61 \%$ la participación de las entrevistadas en el cultivo de papas y ollucos y una mayoría relativa de dichas mujeres realizan los cultivos de granos, como maíz, trigo y quinua. El cultivo de alfalfa y otros forrajes lo realizan principalmente en forma compartida las parejas, 53\% de casos.

En ambas provincias, la crianza del ganado vacuno es compartida por las parejas de agricultores en un promedio de $39 \%$ de casos y en $36 \%$ de casos en la crianza de equinos, apreciándose una relativa mayor participación de los cónyuges. La crianza de porcinos, así como la de aves de corral y de cuyes son actividades que eminentemente corren a cargo de las entrevistadas, en más de $90 \%$ de casos son ellas quienes las realizan.

En general, no existe suficiente conciencia social sobre las contribuciones de la pequeña agricultura familiar y, por lo tanto, no son suficientemente reconocidas ni valoradas en su importancia económica, social, cultural y ambiental. Siguen siendo deficientes las posibilidades a su alcance para acceder al capital (crédito), insumos, servicios de calidad que se ajusten a sus necesidades y que permitan la intensificación sostenible de su producción y rentabilidad.

El trabajo de la mujer en la agricultura alimentaria preserva los conocimientos ancestrales pero también introduce innovaciones y desarrolla formas de trabajo y habilidades. Existe un enorme cúmulo de conocimientos entre los agricultores familiares, en particular de la mujer campesina, aunque poco entendido, valorado, reconocido y difundido por la ciencia, que se expresa, por ejemplo, en el manejo diversificado y altamente flexible de la chacra. No sólo son agricultoras, sino también adaptadoras de tecnologías, creadoras

\section{0/ REVISTA DE SOCIOLOGÍA 25}


y conservadoras de la extraordinaria agro diversidad peruana, fundamento de su gastronomía, una de las más diversificadas y apreciadas del mundo.

Los cambios en los cultivos y en las formas del manejo productivo y comercial, con implicancias en la seguridad alimentaria de los pequeños agricultores de las áreas seleccionadas, permiten apreciar el papel protagonizado por la mujer, como respuesta al boom mediático y del mercado que revalora los productos alimenticios andinos. Mayorías absolutas de 96\% en Quispicanchis y 94\% en La Unión tenían hace 10 años al maíz y a la papa como sus principales cultivos y sólo proporciones muy pequeñas de entrevistadas refirieron que antes cultivaban hortalizas (La Unión) y también trigo y cebada (Quispicanchis). Mayorías similares, 92\% de Quispicanchis y $95 \%$ de La Unión refieren que actualmente sus principales cultivos son las hortalizas y los granos (quinua, kiwicha, tarwi y el maíz). Las mujeres también refieren haber introducido modificaciones de carácter tecnológico en sus cultivos, como la preparación de abonos de corral ( $41 \%$ de casos en Quispicanchis y $46 \%$ en La Unión) y mejoras en la preparación de la tierra ( $27 \%$ de casos en Quispicanchis y $15 \%$ en La Unión), menores porcentajes de ellas refieren haber mejorado las semillas utilizadas por una adecuada selección y de haber mejorado el riego.

Las mujeres explican sus razones para hacer los cambios en sus cultivos y en la forma de cultivar por la necesidad de aumentar su producción, aproximadamente en $51 \%$ de casos en ambas provincias y, en el caso de Quispicanchis, porque la producción resulta de mejor calidad ( $48 \%$ de casos), en tanto que en La Unión explican que es para mejorar el cultivo de kiwicha (29\% de casos).

En lo referente a la persona de la familia que adopta las decisiones para cambiar los cultivos y hacer las mejoras técnicas, se ha encontrado generalmente que en ambas provincias la intervención de la mujer es predominante, así, mientras que en Quispicanchis en 61\% de casos las decisiones las adoptan las parejas en forma mancomunada, en $39 \%$ de casos las adoptan las mujeres y o\% los hombres, en La Unión en $43 \%$ de casos las adoptan las mujeres, en $21 \%$ de casos las adoptan en parejas y $26 \%$ de casos los hombres.

Respecto al acceso a innovaciones en técnicas de cultivo, se encontró que un 90\% de mujeres en Quispicanchis y 65\% en La Unión han aprendido técnicas modernas para mejorar sus cultivos. Por otro lado, un $35 \%$ de mujeres en La Unión y 10\% en Quispicanchis no han aprendido nuevas técnicas para mejorar sus cultivos. Las nuevas técnicas que las mujeres refieren haber aprendido son principalmente, la preparación de abonos (69\% de casos en La Unión y $31 \%$ en Quispicanchis), mejoras en la preparación de la tierra (27\% en Quis- 
picanchis y 8\% en La Unión), adicionalmente en Quispicanchis aprendieron la aplicación de insecticidas (19\% de casos) y la selección de semillas (12\% de casos); refieren así mismo, que recibieron capacitaciones en dichas técnicas y que también las aprendieron de otros agricultores, aproximadamente en similares proporciones ( $47 \%$ de casos). Cerca del $100 \%$ de entrevistadas que han sido encuestadas en Quispicanchis y La Unión aplican en su práctica productiva las técnicas aprendidas en capacitaciones y de otros agricultores, cifra que explica una gran efectividad de los esfuerzos de extensión e innovación entre estas agricultoras.

El 96\% de entrevistadas en Quispicanchis y 69\% en La Unión señalan que la aplicación de las técnicas modernas aprendidas les ha permitido mejorar la calidad de sus cultivos. En La Unión, un $23.1 \%$ señala que las técnicas aplicadas les ha permitido lograr buena producción de kiwicha.

\section{Agricultura alimentaria y mercado}

La notable contribución productiva de la mujer en la agricultura familiar se extiende a la comercialización de sus productos, siendo consistente su participación dada la importancia que asigna a la seguridad alimentaria, complementa y diversifica los alimentos de la canasta familiar y también asegura su economía, para ello programa su calendario productivo en correspondencia con los requerimientos del mercado, los productos demandados, las características y formas de presentación. Las mujeres participan activamente en todas las etapas de la cadena de valor agropecuaria, desde la siembra, cosecha y el proceso de comercialización, en el ejercicio de su función central en la seguridad alimentaria familiar, produciendo alimentos básicos para el consumo del hogar, cuyos excedentes monetiza en los mercados con el fin atender las otras necesidades del hogar.

Como proveedoras de ingresos, las mujeres suplen las necesidades básicas que no pueden ser cubiertas con el producto de la agricultura de subsistencia. En los hogares, las decisiones relativas a la nutrición de la familia, que las mujeres adoptan, tienen que ver con el uso y distribución de los recursos disponibles. Pese a esta participación sustantiva de la mujer en la pequeña agricultura, no se halla equitativamente reconocida ni valorada en los sistemas administrativos ni institucionales. Su trabajo no está debidamente contabilizado en las estadísticas; esta condición de exclusión y la falta de oportunidades de la mujer del campo, son factores que limitan el desarrollo agropecuario y rural, sustentable y equitativo.

\section{2/ REVISTA DE SOCIOLOGÍA 25}


El análisis de las relaciones de los pequeños agricultores productores de alimentos de la sierra sur con el mercado, como los casos estudiados en el presente trabajo, nos revela la relación que existe entre las condiciones de pobreza y el mercado. Es posible apreciar que las productoras más vinculadas al mercado son generalmente menos pobres y, viceversa, las menos vinculadas al mercado son más pobres. A dicho efecto de causalidad se asocian variables que son determinantes, tales como el grado de instrucción de la productora, como principal actora de la pequeña unidad productiva, la tenencia de tierras, el acceso a la tecnología, uso de fertilizantes y la facilidad del acceso al mercado (infraestructura y servicios de transporte).

Los cultivos alimentarios de los pequeños productores de estas provincias los destinan en general a la venta y el autoconsumo. Destaca por su relativa magnitud el destino comercial que dan a la kiwicha, actualmente el principal cultivo en La Unión, producto que lo venden casi en su totalidad, mientras que en Quispicanchis le dan un destino compartido, entre la venta y el autoconsumo. La producción de habas, hortalizas, trigo y cebada destinan preferentemente a la venta y la papa la auto consumen en mayor proporción. El maíz y la quinua principalmente son para la venta en Quispicanchis y en La Unión los auto consumen preferentemente. Su pequeña producción de tarwi ldestinan preferentemente al autoconsumo, sobre todo en La Unión. Ver Tabla 1.

TABLA 1

DESTINO PRINCIPAL DE LOS PRODUCTOS CULTIVADOS, (DISTRIBUCIÓN POR CIENTO)

$\begin{array}{lccc}\begin{array}{l}\text { Destino principal de la } \\ \text { producción de trigo }\end{array} & \begin{array}{c}\text { Provincia } \\ \text { Quispicanchis }\end{array} & \begin{array}{c}\text { Provincia } \\ \text { La Unión }\end{array} & \text { Total } \\ \text { Venta } & 12.5 & \ldots & 10.0 \\ \text { Autoconsumo } & 12.5 & \ldots & 10.0 \\ \text { Venta y autoconsumo } & 75.0 & 100.0 & 80.0 \\ \text { Total } & 100.0 & 100.0 & 100.0 \\ \text { Destino principal de la } & \text { Provincia } & \text { Provincia } & \text { Total } \\ \text { producción de cebada } & \text { Quispicanchis } & \text { La Unión } & \\ \text { Autoconsumo } & \ldots & 66.7 & 25.0 \\ \text { Venta y autoconsumo } & 100.0 & 33.3 & 75.0 \\ \text { Total } & 100.0 & 100.0 & 100.0 \\ \text { Destino principal de la } & \text { Provincia } & \text { Provincia } & \text { Total } \\ \text { producción de papas } & \text { Quispicanchis } & \text { La Unión } & \\ \text { Autoconsumo } & \ldots & 33.3 & 14.3 \\ \text { Venta y autoconsumo } & 100.0 & 66.7 & 85.7 \\ \text { Total } & 100.0 & 100.0 & 100.0\end{array}$




\begin{tabular}{|c|c|c|c|}
\hline $\begin{array}{l}\text { Destino principal de la } \\
\text { producción de habas }\end{array}$ & $\begin{array}{c}\text { Provincia } \\
\text { Quispicanchis }\end{array}$ & $\begin{array}{l}\text { Provincia } \\
\text { La Unión }\end{array}$ & Total \\
\hline Autoconsumo & - & 20.0 & 8.3 \\
\hline Venta y autoconsumo & 100.0 & 80.0 & 91.7 \\
\hline Total & 100.0 & 100.0 & 100.0 \\
\hline $\begin{array}{l}\text { Destino principal de la } \\
\text { producción de quinua }\end{array}$ & $\begin{array}{c}\text { Provincia } \\
\text { Quispicanchis }\end{array}$ & $\begin{array}{l}\text { Provincia } \\
\text { La Unión }\end{array}$ & Total \\
\hline Autoconsumo & 25.0 & 61.5 & 44.0 \\
\hline Venta y autoconsumo & 75.0 & 38.5 & 56.0 \\
\hline Total & 100.0 & 100.0 & 100.0 \\
\hline $\begin{array}{l}\text { Destino principal de la } \\
\text { producción de kiwicha }\end{array}$ & $\begin{array}{c}\text { Provincia } \\
\text { Quispicanchis }\end{array}$ & $\begin{array}{l}\text { Provincia } \\
\text { La Unión }\end{array}$ & Total \\
\hline Venta & 75.0 & 90.0 & 77.8 \\
\hline Venta y autoconsumo & 25.0 & 25.0 & 22.2 \\
\hline Total & 100.0 & 100.0 & 100.0 \\
\hline $\begin{array}{l}\text { Destino principal de la } \\
\text { producción de tarwi }\end{array}$ & $\begin{array}{c}\text { Provincia } \\
\text { Quispicanchis }\end{array}$ & $\begin{array}{l}\text { Provincia } \\
\text { La Unión }\end{array}$ & Total \\
\hline Autoconsumo & 40.0 & 66.7 & 54.5 \\
\hline Venta y autoconsumo & 60.0 & $33 \cdot 3$ & $45 \cdot 5$ \\
\hline Total & 100.0 & 100.0 & 100.0 \\
\hline $\begin{array}{l}\text { Destino principal de la } \\
\text { producción de hortalizas }\end{array}$ & $\begin{array}{c}\text { Provincia } \\
\text { Quispicanchis }\end{array}$ & $\begin{array}{l}\text { Provincia } \\
\text { La Unión }\end{array}$ & Total \\
\hline Autoconsumo & 25.0 & 66.7 & 34.6 \\
\hline Venta y autoconsumo & 75.0 & $33 \cdot 3$ & 65.4 \\
\hline Total & 100.0 & 100.0 & 100.0 \\
\hline $\begin{array}{l}\text { Destino principal de la } \\
\text { producción de maíz }\end{array}$ & $\begin{array}{c}\text { Provincia } \\
\text { Quispicanchis }\end{array}$ & $\begin{array}{l}\text { Provincia } \\
\text { La Unión }\end{array}$ & Total \\
\hline Autoconsumo & 25.0 & 61.5 & 44.0 \\
\hline Venta y autoconsumo & 75.0 & 38.5 & 56.0 \\
\hline Total & 100.0 & 100.0 & 100.0 \\
\hline
\end{tabular}

Fuente: Encuesta Socioeconómica. Marzo 2015.

La preparación y procesamiento de los productos agrícolas de la unidad familiar para su venta es realizada preponderantemente por la mujer en ambas provincias, así, encontramos que la preparación de hortalizas, verduras y flores la realizan en $93 \%$ de casos las entrevistadas, menestras en $90 \%$ de casos, y tubérculos en $69 \%$ de casos. La preparación de los granos, como maíz, trigo, cebada, kiwicha y quinua, así como de alfalfa y otros forrajes las realizan en forma compartida entre las parejas, aunque siempre con una relativa mayor presencia de la mujer.

En general, las mujeres de estas familias de pequeños productores tienen una mayor participación activa en la venta de sus productos. Encontramos así 
que en amplias mayorías, entre $81 \%$ y $98 \%$ de casos, son las mujeres quienes hacen la venta de hortalizas/verduras/flores y menestras (habas, arvejas), así como la venta de animales menores vivos y beneficiados. Son mayorías de casos, entre $52 \%$ y $67 \%$ en los que también la mujer se encarga de vender los granos (maíz, quinua, trigo y kiwicha) y de tubérculos (principalmente papas). Los cónyuges se encargan en un promedio de $56 \%$ de casos, en ambas provincias, de la venta de carnes de animales mayores. En todos los casos de venta de los diversos productos, se agrega la participación mancomunada de la mujer y el cónyuge (entre $2.5 \%$ y $37.5 \%$ de casos).

El transporte y venta de los productos de alfalfa y otros forrajes, según refieren las entrevistadas, realizan en $71 \%$ de casos las parejas de agricultores, en forma conjunta, siendo más acentuada ( $86 \%$ de casos) esta tendencia en la provincia de Quispicanchis, mientras que en La Unión es perceptible una mayor presencia de la mujer (39\% de casos).

El miembro de familia que lleva a cabo el transporte y venta de productos pecuarios como ganado en píe, en $64 \%$ de los casos lo realiza el cónyuge solo, siendo algo mayor esta tendencia en Quispicanchis y en $27 \%$ de casos lo hacen las parejas. En la provincia de la Unión es la mujer en un $40 \%$ de unidades familiares que realiza dichas labores. El transporte y venta de animales menores vivos como también de animales menores beneficiados (carne) los realizan las entrevistadas de ambas provincias en $97 \%$ de casos y en $3 \%$ los hacen conjuntamente con el cónyuge.

En el último decenio, la población agrícola rural se ha visto afectada de manera directa y creciente por nuevos procesos, como resultado del contexto mundial globalizado y la liberalización del comercio en rápida evolución. Los pequeños agricultores enfrentan condiciones crecientemente dominadas por el mercado, más dinámicas y cambiantes y menos previsibles. Este contexto de cambios ha traído consigo el boom que revalora los productos andinos, coyuntura novedosa que la mujer campesina capta con agudeza e implementa en sus sistemas de pequeña productora de alimentos las adaptaciones que el mercado demanda, acorde a la importancia que actualmente se asigna a los granos andinos, como la quinua y kiwicha, a los tubérculos y otros, por sus cualidades nutritivas y ecológicas.

Las mujeres campesinas de las áreas objeto de estudio se mantienen informadas de la situación y perspectivas de los mercados locales y regionales y son innovadoras en los circuitos de comercialización de los productos alimenticios, conocen, adecuan su actividad comercial a la par que sustituyen cultivos, en razón a la demanda. Se encontró que un 92\% de las mujeres de las 
dos provincias saben que en los últimos años se está dando en las ciudades mayor importancia a los granos como la quinua, kiwicha, tarwi y maíz. Un 8\% de mujeres manifestó no saberlo.

En Quispicanchis y en La Unión un 86\% de las mujeres se han informado en el mercado de la importancia y valor que actualmente se asigna a los granos y productos de la sierra. Cabe recordar que las pequeñas agricultoras andinas actualmente mantienen estrecho contacto comercial con los mercados regionales, lo que les permite orientar su actividad productiva. Las amistades, la televisión y la radio contribuyen a divulgar dicho conocimiento. La totalidad de mujeres en la provincia de La Unión y $89 \%$ de Quispicanchis han percibido que en los últimos años hay más compradores que demandan los granos y productos andinos. Una amplia mayoría de $84 \%$ de ellas señala que los precios de los mismos han subido, un $9 . \%$ de señalan que han bajado y un $7 \%$ de ellas que los precios siguen igual.

En La Unión y Quispicanchis casi la totalidad de mujeres considera importante que en el país y el mundo se esté dando más valor a los productos agrícolas de la sierra como los que cultiva, respuesta sumamente significativa que refleja el efecto motivador que se estaría dando en la conciencia individual y colectiva de las mujeres de las pequeñas unidades productoras de alimentos, empoderándolas en sus propósitos de innovar sus formas de producción, rescatando los recursos culturales tradicionales,

Una mayoría de $52 \%$ de las mujeres explica que la razón por la cual estiman importante el mayor valor que actualmente se asigna a los productos agrícolas de la sierra es porque ello les permite mejorar su situación económica, adicionalmente, un $17 \%$ de las mismas lo explican porque dichos granos (quinua, kiwicha, tarwi y el maíz) tienen un alto valor nutritivo. Esta tendencia se repite por provincias.

La comercialización de productos realizada por las mujeres, favorece las relaciones sociales y la cohesión social entre las comunidades, en el caso de las mujeres en estudio, viajan para vender sus productos y también recolectan de otros campesinos, prestándose ayuda mutua, con hortalizas, flores y frutos, incluso decoran sus paquetes con los productos más codiciados y vendibles. Dicho comportamiento nos conduce a distinguir la emergencia de una definida cultura de mercado a pequeña escala, que se hace más visible en la provincia de Quispicanchis. Al margen de las diferencias económicas entre las mujeres pequeñas productoras, su actitud orientada hacia el mercado es generalizada y se conduce bajo formas solidarias de cooperación.

\section{6/ ReVISTA de SOCIOLOGÍA 25}


En estos aspectos, las mujeres se encargan de procesos que contribuyen en la economía agrícola familiar a partir de la mejora de los productos agropecuarios, tal como se ve en las experiencias del cultivo de hortalizas y flores y en la crianza de cuyes en forma organizada, en la comunidad de Quispicanchis, así como la introducción del cultivo de kiwicha y las nuevas formas de comercialización que actualmente se aplican en La Unión. Dichas innovaciones responden a campañas de capacitación técnica operadas por organismos externos, que las mujeres campesinas capitalizan tratando de mejorar la calidad y cantidad de sus productos, en condiciones adaptadas a la demanda de los mercados que conocen y cuya venta les ofrece la posibilidad de mejorar sus ingresos, así como el autoconsumo familiar.

Dichas experiencias no agregan nuevas tareas a la mujer sino que se complementan con su laboreo cotidiano, con la inicial capacitación recibida buscan incrementar sus rendimientos productivos y los ingresos por su venta en ferias y mercados regionales e incluso destinan a la exportación, como en el caso de la kiwicha, que la ofrecen clasificada y envasada.

Las mejoras en los ingresos por la actividad innovadora de la mujer son destinadas a necesidades reales de las familias, como la educación, salud y alimentación. Estas experiencias confirman estudios que revelan la asociación entre mejoras alimentarias y el acceso de las mujeres a ingresos propios y tal como lo plantea $\mathrm{H}$. Fuentes, las intervenciones en seguridad alimentaria pública y privada tienen el enorme desafío de reorientar sus lineamientos para asegurar el tratamiento de los problemas que limitan el papel de la mujer en la seguridad alimentaria, toda vez que al ejercer el control directo de los ingresos, las mujeres tienden a invertirlos en el bienestar de la familia $y$, sobre todo, en la mejora de su seguridad alimentaria. ${ }^{14}$

Es perceptible que se viene dando cierta autonomía económica de las mujeres campesinas como respuesta a su propio impulso, al incursionar en actividades productivas de pequeños negocios, con un nivel de organización asociativo, como se da en los casos de la crianza de cuyes en forma tecnificada en las comunidades de Quispicanchis y las asociaciones de productores de kiwicha de exportación, en la provincia de La Unión. Estas actividades pueden ser consideradas estratégicas, ya que las mujeres que las adoptan pueden generar sus propios ingresos en actividades productivas y de esa manera lograr autonomía, empoderamiento y contribuir a mejorar la seguridad alimentaria familiar. 
Un caso concreto de este tipo de negocios es la crianza de cuyes en la provincia de Quispicanchis, un producto tradicional en la región cuya producción y comercialización han cambiado la geografía económica de las comunidades, por cuanto, esta tarea que corre a cargo de la mujer genera ingresos nuevos y contribuye en la alimentación familiar. La crianza de cuyes ha generado nuevas formas de organización entre las mujeres, quienes se han asociado para compartir servicios en el manejo de esta crianza, tal el caso de un grupo de mujeres que en promedio tienen cada una cuatrocientos cuyes. Dicha experiencia muestra que para desarrollar una pequeña empresa se requiere contar con algunos activos productivos propios, una demostrada capacidad de innovación, capital humano y una lógica empresarial competitiva. El factor organizativo, los valores solidarios y la democratización de los aprendizajes y técnicas, juegan un papel importante en el éxito de este tipo de negocio, conducido por mujeres. Estas experiencias han sido logradas al haberse adoptado técnicas de crianza mejoradas, con productos de calidad, mejor cotizados en el mercado y que adicionalmente, contribuyen a mejorar la alimentación familiar de las productoras.

Las formas de comercialización son diversas, llegan al lugar compradores en tiempos previstos y por determinadas cantidades, también las mujeres venden directamente en mercados regionales y en la ciudad del Cusco, así mismo, ofrecen en el mercado productos con valor agregado, preparando platos a base de cuyes, que venden en los mercados de la región. Esta actividad desarrollada por las mujeres en Quispicanchis ha alcanzado éxito económico y de prestigio, en concursos regionales de promoción económica y social.

Respecto a los cambios en las formas y estrategias adoptadas por las entrevistadas para comercializar sus productos, se ha encontrado que cerca del 76\% en Quispicanchis y La Unión han introducido cambios en la preparación y procesamiento de sus productos para la venta, sin embargo, una cuarta parte de ellas refiere no haber hecho cambios. Los principales cambios adoptados son, limpiar, ordenar, seleccionar los productos de calidad y presentar mejor los productos. Las decisiones sobre dichos cambios han sido adoptadas por las mujeres en una mayoría promedio de $77 \%$ de casos en ambas provincias.

Un 86\% de entrevistadas en Quispicanchis y 100\% en La Unión han adoptado cambios en la composición de su oferta de venta de productos agrícolas, siendo más notorias las adoptadas en la provincia de La Unión, donde hace diez años el producto más vendido era el maíz, tanto sólo como asociado con quinua, también con tubérculos, con forraje y con hortalizas en $54 \%$ de los casos, en segundo orden de importancia vendían entonces papas y otros

\section{8/ REVISTA DE SOCIOLOGÍA 25}


tubérculos en $19 \%$ de los casos, mientras que en la actualidad el producto que más venden es la kiwicha, mayormente sola y en pequeña proporción asociada con quinua y también con tarwi en $63 \%$ de los casos, sectores más pequeños $16 \%$ de entrevistas y $10.5 \%$ de las mismas venden ahora tubérculos, asociados con quinua y con maíz.

En Quispicanchis se han dado cambios importantes en la composición de la oferta de venta de las mujeres, dado que hace diez años el producto más vendido era el maíz, tanto sólo, como asociado con quinua, con tubérculos, con forraje y también con hortalizas, totalizando prácticamente el $100 \%$ de casos y actualmente venden en mayor proporción hortalizas, generalmente asociadas con maíz, con quinua y también con flores, en $65 \%$ de casos, siguiéndoles las ventas de maíz, asociado con hortalizas y con quinua, en $61.5 \%$ de los casos, siendo también significativos los sectores que venden kiwicha, quinua y tarwi, en $19 \%$ de casos.

El dinamismo comercial que adoptan las pequeñas productoras de alimentos de estas provincias se aprecia también por los cambios de los mercados a los que acceden para vender sus productos, generalmente en búsqueda de maximizar sus ingresos. En el caso de las encuestadas de Quispicanchis se encontró que hace diez años un $71 \%$ de mujeres vendía sus productos en el mercado local y/o provincial (Urcos), un $18 \%$ de las mismas vendía en un departamento vecino (Puno) y un $7 \%$ vendía en la capital de departamento (Cusco). Actualmente, un $60 \%$ de dichas mujeres refieren que venden en mercados de los departamentos vecinos (principalmente en Puno y también en Arequipa), un sector minoritario de $12 \%$ de encuestadas refiere vender actualmente sus productos en la capital del departamento (Cusco) y un escaso $4 \%$ lo hace en la capital provincial (Urcos). La totalidad de encuestadas de La Unión reseñan que hace diez años vendían sus productos en el mercado local y provincial, mientras que ahora $70 \%$ de las mismas venden sus productos en la capital de departamento (Arequipa) y sólo un 30\% de ellas venden en los mercados local y provincial. Se distingue en esta provincia la importancia que ha adquirido el comercio de la kiwicha, para cuyas transacciones acuden a los predios agrícolas rescatistas vinculados a la exportación.

Un aspecto que también denota cambios importantes en la actividad comercial de las pequeñas productoras de alimentos de la provincia de Quispicanchis es la frecuencia con que salen a vender a los mercados. Hace diez años una notable mayoría de $77 \%$ de encuestadas vendía una vez por semana, $14 \%$ de las mismas lo hacía cada mes y $9 \%$ dos veces por semana. Al presente, se aprecia un notable incremento en la frecuencia de esas ventas, ya que 
un $57 \%$ de encuestadas vende tres veces por semana, $14 \%$ de las mismas lo hace una vez por semana y $21 \%$ cada mes. En Quispicanchis, según refieren las encuestadas, se han incrementado cerca de $40 \%$ tanto las cantidades vendidas de sus productos como los precios de los mismos.

Mayorías cercanas al 60\% de encuestadas de Quispicanchis y de La Unión refieren que son ellas quienes deciden hacer los cambios en la venta de sus productos, menores proporciones de ellas 38\% en Quispicanchis y $25 \%$ en la Unión, refieren que son decisiones compartidas con el cónyuge.

Respecto a la variación en los ingresos familiares por la venta de sus productos, $77 \%$ de las mujeres en Quispicanchis y 55\% de La Unión refieren que sus ganancias por la venta de sus productos han mejorado regularmente en los últimos años. Proporciones de $23 \%$ en Quispicanchis y $10 \%$ en La Unión refieren que sus ganancias han mejorado bastante. Del total de Encuestadas que señalan que han mejorado regularmente sus ganancias, en $55 \%$ de casos en Quispicanchis lo consideran así porque han mejorado sus productos, mientras que en $46 \%$ de casos en La Unión lo explican porque no se gana mucho en esos productos. Del total que señalan que han mejorado bastante sus ganancias, la totalidad en Quispicanchis lo consideran así porque han mejorado la calidad de sus productos, similarmente, casi todas las mujeres en La Unión refieren que la kiwicha genera buena ganancia. Del total que señalan que han mejorado poco sus ganancias, 33\% de las mujeres Encuestadas de La Unión lo consideran así porque no se gana mucho y otro $33 \%$ porque sus terrenos son pequeños.

\section{Seguridad alimentaria y estrategias agropecuarias}

Consideramos pertinente profundizar el conocimiento sobre las percepciones que conducen a las mujeres campesinas en su accionar agropecuario, en el pequeño comercio que practican, en su capacidad de transformación e innovación productiva y de guardianas de la seguridad alimentaria familiar que, como se ha expuesto, las perfilan como agentes de transformación estructural de la agricultura familiar, generando nuevas oportunidades productivas y comerciales, competitivas en mercados regionales y externos, así mismo, consideramos necesario indagar cómo afrontan las situaciones que atentan contra la seguridad alimentaria y el bienestar familiar, frecuentes entre las familias más pobres, por efecto de los fenómenos climáticos anormales y sus secuelas (sequías, inundaciones, etc) y/o por fluctuaciones del mercado cambiante, que merman severamente sus ingresos.

\section{0/ REVISTA DE SOCIOLOGÍA 25}


La soberanía alimentaria en la pequeña agricultura andina se sustenta en modos de producción local y prácticas de desarrollo sostenible. Además de los sistemas de cultivo de riego y de secano, en los que la participación de las mujeres es fundamental, son de especial importancia el manejo de los huertos familiares, ubicados en los espacios adyacentes a las viviendas rurales, así como la crianza de animales, que tradicionalmente contribuyen a la alimentación de la familia campesina y a la generación de ingresos.

Entre las estrategias que adoptan las mujeres se distingue la agricultura del huerto familiar, ámbito en el que diversifican las especies cultivadas, con hortalizas, frutos y otros alimentos básicos que contribuyen a la seguridad alimentaria familiar. Así mismo, los excedentes de los alimentos producidos en el huerto son comercializados en los mercados locales, posibilitando captar ingresos complementarios para la dieta. El huerto es un sistema productivo de policultivos, donde las hortalizas, verduras, flores y árboles frutales son predominantes, lo que permite que las familias dispongan de dichos productos, que generalmente no se obtienen del mercado.

En la zona sur andina del país, los huertos son multifuncionales y producen alimentos de manera sustentable y ecológica. Así mismo, cumplen funciones sociales importantes para el esparcimiento familiar, el ornato y la ecología, contribuyen a la conservación de suelos, la generación de microclimas y la biodiversidad. La producción del huerto no sólo es fuente de una cantidad importante de alimentos sino también de productos medicinales, es capaz de absorber la mano de obra familiar eventualmente excedente y proveer los insumos mínimos necesarios para la supervivencia de los miembros de la familia. Es el espacio de las viviendas que ofrece sombra, cortina rompe vientos y cerco vivo. Proporciona también alimentos para los animales de corral (cuyes y gallinas) y hasta hábitat para la fauna silvestre. Para las familias campesinas, los huertos tienen un "uso versátil”. ${ }^{15}$

La diversidad biológica de los huertos es amplia y guarda relación con la participación efectiva de la mujer, generadora de conocimiento hortícola y alimentario, quien ensaya la adaptación de plantas silvestres medicinales y la obtención de semillas, flores, follaje, frutos y tallos. La experimentación por parte de las mujeres, mediante el cultivo de nuevas plantas al interior y en los límites del huerto es importante. La propagación de especies vegetales en huertos vecinos es parte del intercambio practicado por las mujeres, cuando se percatan de la existencia de alguna especie o variedad novedosa, con fruto o

Pérez, José y Gutierrez, Gastón. Revista de Agroecología. México. 2009. 
parte comestible y que puede ser vendido en el mercado. Los huertos poseen "diversidad estructural". ${ }^{16}$ Las campesinas también cultivan plantas que no son propias de la zona, pero que mediante cuidados logran adaptarlas. Así, en Quispicanchis, están adaptando rosas importadas, logrando reproducirlas con buena calidad para su venta. Algunas han logrado instalar fito toldos, con asistencia técnica para el propósito.

Algunas mujeres entrevistadas de La Unión refieren que procesan el secado de frutos, semillas y hojas, tal como orégano, semillas de calabaza, rocotos y frutos, que en parte los reciclan como semillas. Las mujeres del sur andino participan tanto en la agricultura alimentaria como en el huerto, demuestran una gran capacidad de innovación, tanto en el manejo de plantas comestibles como la kiwicha en La Unión y de frutas de temporada y flores en Quispicanchis, donde han generado nuevas oportunidades de desarrollo y seguridad alimentaria para sus familias. Se suma a ello el manejo de cultivos medicinales, como fuente temporal de ingreso familiar. ${ }^{17}$

Una amplia mayoría de $83.0 \%$ de las Encuestadas de ambas provincias tienen un huerto anexo a su vivienda, dicha proporción es algo mayor en Quispicanchis. El tamaño promedio de los huertos es de 0.03 Has., siendo algo más grandes en la provincia de La Unión. En ambas provincias el manejo de los huertos, a cargo de las entrevistadas, comprende cultivos asociados de hortalizas, con verduras y flores ( $65 \%$ de casos), siguiéndole en importancia las huertas de hortalizas con frutales ( $29 \%$ de casos), sobre todo en Quispicanchis y en menores proporciones las de sólo frutales (Quispicanchis) y las de Menestras con habas, frijoles y lentejas (La Unión).

Se observa que casi la tercera parte de los productos cultivados en huertos son destinados exclusivamente para la venta, un $10 \%$ se destina para el autoconsumo y $61.5 \%$ se destina tanto para la venta como para el autoconsumo.

Entre las estrategias que las mujeres campesinas ponen en práctica para enfrentar sus dificultades destaca su permanencia como productoras en el predio familiar incluido el manejo del huerto y la crianza de animales de especies menores, actividades que en algunos casos han adquirido categoría de pequeños negocios, como se puede apreciar en los puntos anteriormente expuestos del presente artículo. Las mujeres campesinas pobres de ambas provincias recurren también a otras estrategias, como la de contratarse en trabajos agrícolas a tiempo parcial, en labores de cosecha, lavado y empaque de hortalizas, riego, cosecha, clasificación y empaque de la kiwicha, entre

16 Gonzales, J.A. Cultura y Agricultura: Transformación en el Agro Mexicano. México. 2003.

17 Ballara, M., Parada, S. FAO. 2007.

\section{2/ REVISTA DE SOCIOLOGÍA 25}


otras. La retribución obtenida la dedican en parte a la adquisición de alimentos para su familia, también se dan casos en que las compensaciones del trabajo se dan en alimentos. Recurren así mismo, particularmente las mujeres más pobres, a la preparación y venta ambulatoria de platos de comida, utilizando sus propios insumos, generalmente en los mercados locales.

Es remarcable que este tipo de actividades en la agricultura familiar se dan como parte de trabajo no remunerado y son "invisibles" a niveles institucionales y administrativos, concurriendo al conjunto de inequidades que pesan todavía en las desigualdades que afrontan las mujeres campesinas, manteniendo la trama de atraso y pobreza en los sectores rurales dedicados a la agricultura.

Siendo las mujeres campesinas quienes asumen en las pequeñas unidades familiares la responsabilidad de la seguridad alimentaria, analizamos a continuación la racionalidad con la que conducen el destino de sus recursos familiares, productos de cosechas, animales de crianza que benefician y los ingresos de su monetización en los mercados. Encontramos que sectores mayoritarios de las mujeres encuestadas, 55\% de Quispicanchis y $75 \%$ de La Unión, consideran que la forma más acertada para asegurar el bienestar de su familia es principalmente vender sus productos y con sus ganancias comprar los alimentos faltantes. Consecuentemente, proporciones menores, $45 \%$ en Quispicanchis y $25 \%$ de encuestadas en La Unión consideran que es más acertado consumir preferentemente los productos que cultivan y los sobrantes venderlos para comprar lo que falta de alimentos y otros bienes necesarios.

Del total de encuestadas que consideran que la forma más acertada para asegurar el bienestar de su familia es vender sus productos y con la ganancia comprar los alimentos, justifican tal decisión porque de los ingresos subsiste la familia y también afirman que se cosecha para vender y consumir y que las ganancias les permite comprar otros alimentos y bienes faltantes.

Del las encuestadas que consideran que la forma más acertada para asegurar el bienestar de su familia es consumir los productos que cultivan y los sobrantes venderlos para comprar lo que falta de alimentos y otros bienes necesarios, en Quispicanchis la justifican porque es mejor la alimentación natural ( $42 \%$ de casos) y porque se cosecha para vender y consumir $33 \%$ de casos), mientras que en La Unión refieren que la producción no alcanza para vender (60\% de casos) y que es mejor la alimentación natural (40\%de casos).

En relación a la participación de los miembros de familia en la adopción de actividades de subsistencia para afrontar necesidades económicas graves o imprevistas, se encontró en ambas provincias que un $95 \%$ de las mujeres 
encuestadas de ambas provincias señalan que venden las hortalizas de sus cultivos de huerta; el 90\% de mujeres vende los animales de su crianza, $92 \%$ de ellas vende comida como ambulante y un $64 \%$ de las mismas se contrata como asalariada. La alternativa de migrar para asalariarse es adoptada en $79 \%$ de los casos por los cónyuges de ambas provincias aunque es significativa la proporción (40\%) de mujeres de la provincia de La Unión que también migran con tal propósito.

La composición y los cambios operados en la canasta alimentaria familiar de los pequeños agricultores, relativos al consumo de sus propios cultivos, frente al consumo de productos industriales sucedáneos, presenta el panorama descrito a continuación. Una mayoría de $69 \%$ de las mujeres encuestadas en Quispicanchis refieren emplear más que antes sus cultivos cosechados en la alimentación de su familia, en cambio, un $55 \%$ en La Unión afirman que los emplean igual que antes. Del total de encuestadas que señalan emplear sus cultivos más que antes en la alimentación de su familia, un 50\% en Quispicanchis lo justifican porque la calidad de la producción es buena y $33 \%$ de La Unión porque la producción es para el autoconsumo porque en las ventas se gana poco. De las mujeres que señalan emplear sus cultivos igual que antes en la alimentación de su familia, un 50\% en Quispicanchis lo considera así porque la calidad de la producción es buena, en cambio, 33\% en La Unión porque la producción es para el autoconsumo y que en las ventas se gana poco.

Los hábitos alimentarios que la mujer adopta respecto al uso de los insumos a los que recurre en la preparación de los alimentos familiaraes, muestran los siguientes comportamientos:

Prácticamente la totalidad de las encuestadas de las dos provincias emplean todos los días papas.

Una gran mayoría de $73 \%$ de mujeres encuestadas de ambas provincias tienen preferencia por el consumo diario de cereales y derivados industriales, como arroz, fideos y tallarines, mientras que el $27 \%$ restante de dichas mujeres consumen estos cereales y sus derivados unas tres veces por semana.

Proporciones relativamente mayoritarias de las encuestadas (entre $51 \%$ y $62 \%$ de casos) de La Unión y Quispicanchis refieren consumir diariamente los granos de su propia producción (principalmente maíz y habas). También refieren consumir regularmente sus producciones de trigo, cebada, arvejas, quinua y ollucos. Son menos frecuentes los casos que consumen kiwicha y tarwi.

Es relevante mencionar que el consumo diario de carnes se da aproximadamente en un $29 \%$ de casos en ambas provincias, otro $26 \%$ de casos que

\section{4/ REVISTA DE SOCIOLOGÍA 25}


consumen carne unas tres veces por semana y un $37 \%$ de casos que pocas veces consumen carnes.

Sectores mayoritarios de las mujeres encuestadas, entre $74 \%$ de casos y 65\% de casos, en Quispicanchis y La Unión coinciden en explicar sus preferencias por emplear arroz, fideos y tallarines en la preparación de los alimentos de su familia, en razón a la facilidad de su preparación; adicionalmente sectores de las entrevistadas señalan que los fideos y tallarines cuecen rápido. Las preferencias del consuno de los productos industriales referidos por parte de las mujeres pequeñas agricultoras, que apuntan a la facilidad y rapidez de la preparación de los alimentos familiares con tales insumos, tendrían que ver, entre otros factores, con la acuciante presión de horas de trabajo diario de la mujer campesina.

Mayorías notables de entrevistadas, entre 63\% y 77\% de casos, en Quispicanchis y La Unión, respectivamente, consideran que el principal inconveniente para emplear maíz, quinua, kiwicha y papas en la preparación de los alimentos familiares, es su precio alto y que por lo tanto es mejor venderlos; también son notables las proporciones de mujeres (entre 50\% y 56\% de casos) de las dos provincias que similarmente afirman respecto a las habas, arvejas y tarwi que prefieren venderlos por su mejor precio en el mercado y que, además, es más difícil su preparación para la alimentación familiar. Una mayoría de $67 \%$ de entrevistadas refieren que preparar el olluco es más difícil, por lo que prefieren venderlo y una apreciación similar, aunque de sectores algo menores de mujeres, se refieren al empleo del trigo y cebada que ellas producen.

\section{Conclusiones}

Las mujeres campesinas son reproductoras y cultivadoras de la agro diversidad andina, pero también son exploradoras de nuevas alternativas y experimentan empíricamente, adaptando especies y variedades en la producción de sus predios familiares. Dicho papel de las mujeres en las pequeñas unidades agropecuarias las ha posicionado últimamente como las verdaderas impulsoras de los cambios operados en la producción y comercialización de los alimentos que consumen los peruanos, no obstante que ellas todavía enfrentan inequidades sociales, económicas, políticas y socioculturales.

La mujer campesina de la región sur andina peruana, cumple un rol social de gran importancia pues su participación es efectiva y, además está en capacidad de generar efectos de carácter multiplicador en la familia, la comunidad y la región; siempre y cuando se reconozca su aporte al conocimiento y a la 
economía familiar. Todavía sigue pendiente la maduración social que lleve a reconocer y respetar los derechos de la mujer y su papel fundamental en la producción de alimentos para la familia por cuanto ella es protagonista principal, aunque anónima, en los propósitos de soberanía alimentaria del país, tarea que todavía está pendiente, dado que se desenvuelve restringida por limitaciones socioeconómicas, que se manifiestan paradójicamente, por ejemplo, en no poder aprovechar directamente las bondades nutritivas de sus propios productos en la alimentación de su familia, por atender sus necesidades de monetización.

La presente investigación contribuye a explicar desde la óptica y racionalidad de la mujer campesina el impacto en los pequeños productores agrarios y las respuestas de éstos frente al boom que revalora los productos alimenticios andinos, habiéndose encontrado que es la mujer, por su mayor cercanía a los mercados locales y regionales, quien avizora las oportunidades y formas que le permiten introducir innovaciones y otros cambios en la producción agrícola, la preparación de los productos y su comercialización, habiendo modificado así su portafolio de cultivos, con mayor presencia de productos andinos tales como quinua y kiwicha, productos rápidamente monetizables, como hortalizas y flores, así como al haber tecnificado e innovado a escala comercial la crianza de cuyes.

El análisis del manejo de la seguridad alimentaria familiar de los pequeños productores agrarios, que corre a cargo de la mujer madre de familia, visibiliza el dilema que afronta la mujer, en la disyuntiva de sus precariedades económicas, de monetizar sus productos agrícolas de mejores propiedades nutritivas, pero mejor cotizados en el mercado, o destinarlos directamente a la alimentación de su familia. Los resultados obtenidos muestran que en los años recientes se han incorporado parcialmente los productos andinos en la dieta alimentaria de un sector de pequeños agricultores, en las dos provincias en estudio.

Las mujeres de las pequeñas unidades agropecuarias han tomado conocimiento en los últimos años de la importancia que cobran sus productos agrícolas tradicionales, principalmente por la mayor demanda de éstos en los mercados. Los cambios en el portafolio de cultivos, en la oferta comercial de dichos agricultores y en las estrategias alimentarias, tienen que ver con sus aptitudes productivas, la disponibilidad de sus recursos materiales y tecnológicos y con sus necesidades de monetización.

La inseguridad alimentaria es uno de los grandes problemas que afecta a las zonas rurales, particularmente en la sierra del Perú y se manifiesta en los altos índices de desnutrición infantil. Esta situación tiene entre sus con-

\section{6/ REVISTA DE SOC1OLOGíA 25}


dicionantes las restricciones socio económicas y culturales inequitativas que atentan contra la formación y el reconocimiento de la mujer, como agente responsable de las unidades agropecuarias que conduce.

\section{Referencias bibliográficas}

Ballara, M. y Damianovic, N. (2010). Políticas para fortalecer la contribución de las mujeres a la agricultura y la seguridad alimentaria. Lima: Instituto Interamericano de Cooperación para la Agricultura - IICA.

BALLARA, Marcela (2009). La mujer en la agricultura, medio ambiente y la producción rural. Chile: FAO.

Heller. L. (2010). Mujeres emprendedoras en América latina y El Caribe: Realidades, obstáculos y desafíos. Chile: CEPAL, Serie Mujer y Desarrollo № 93.

GarCía FríAs, Z. (2006). Equidad de género y agricultura. Su importancia en una época de globalización económica. En Voletín SD Dimensiones. FAO.

DESCO (2010). Programa Selva Central: Plan de mejora. Lima.

FAO (2000). Mejorando la nutrición a través de huertos y granjas familiares. Manual de capacitación para trabajadores de campo en América Latina. Roma: FAO.

Berrio, A., Ć́rdenAs, S. y ZuloagA, G. (2009). Cosecha de aprendizajes: Experiencia de mujeres para la soberanía alimentaria. Lima.

Eguren, F. (2011). Seguridad Alimentaria. SEPIA XIV. Piura, Perú.

SAENGER, C. (2001). Equidad y género y agricultura sostenible. Lima: Ed. F. Tristán.

LOAYZA, S. (2006). Cambios en las relaciones de trabajo de la mujer en comunidades campesinas. Investigaciones Sociales 17. Lima: IIHS-UNMSM.

KUAN, J. (2009). La crisis alimentaria: Retos y oportunidades en los Andes. En Contribuciones para el desarrollo sostenible en los Andes. CONDESAN No. 9. Santiago de Chile: FAO. 